\title{
Automobile Speed Violation Detection System using RFID and GSM Technologies
}

\author{
Lujaina Al-Shabibi \\ Student, Telecommunications \\ Engineering \\ Caledonian College of \\ Engineering Muscat, Oman
}

\author{
Nadarajan Jayaraman \\ Senior Lecturer \\ Caledonian College of \\ Engineering \\ Muscat, Oman
}

\author{
Jayavrinda Vrindavanam \\ Senior Lecturer \\ Caledonian College of \\ Engineering \\ Muscat, Oman
}

\begin{abstract}
The intention to work on the topic of this nature is emanated from the interest to explore alternative technological solution to achieve effective speed control. The objective of the study is to design and develop a new system that can effectively detect speed violations on the road and supports the driver to obey traffic rules while driving by maintaining the speed according to the speed limit prescribed. Based on the detailed review of the literature and also based on the comparison among the prevailing approaches in addressing the core issues, the study has introduced an efficient system with an enhanced scope. The proposed system consists of PIC (18F45K22) microcontroller, Radio Frequency Identification (RFID) and Global System for Mobile Communications (GSM). The system is having two RFID readers along with a passive tag that is attached to the vehicle and GSM is utilized to notify the vehicle's owner and Police through Short Message Service (SMS). Furthermore, a picture of a vehicle is taken via the Camera and a fine is charged when the speed limit is exceeded. The system offers low cost, reliable, efficient results and real time notification. The system was designed and simulated using Proteus v7.10 and the system was operating successfully and performed accurately.
\end{abstract}

\section{General Terms}

Radio Frequency Identification (RFID), Global System for Mobile Communications (GSM).

\section{Keywords}

Detecting Speed, Global System for Mobile Communications (GSM), Radio Frequency Identification (RFID), PIC (18F45K22) Microcontroller, Passive Tag, Traffic Rules, Camera.

\section{INTRODUCTION}

With the progress of time, the society uses improved technology in almost everything as it has enhanced our life style and added benefits. Throughout the earlier years many devices and technologies has been utilized to provide road safety and accordingly to reduce accidents occurring due to speed violation, for example: RADAR technology, PoliScan Speed systems, Average Speed Safety Camera, etc. This paper presents an automobile speed violation detecting system using Global System for Mobile Communications (GSM) technology and Radio Frequency Identification (RFID) technology, as a solution to the general problem of road speed violations and related accidents on the roads. The idea is to design a system that can be employed to detect any speed violation occurring on the road and then send SMS to both the owner of the car and the Police, also take a snap using the following components RFID readers, camera and GSM modem linked to microcontroller (PIC18F45K22). The key factor of selecting RFID technology as a core component of the proposed system is to proposing a cost effective, accurate, flexible system to help in preventing over speeding violations and to reduce fatal accidents. The rest of the paper is organized as follows: Section 2 presents a set of recent literatures that has been carried out, Section 3 is on the design and the framework of the proposed system, Section 4 provides design simulation with results and Section 5 covers the conclusions.

\section{LITERATURE REVIEW}

Hiasat \& Almomani [1], designed and implemented a system that integrates Radio Frequency Identification (RFID) along with Global System for Mobile Communications (GSM) to manage the traffic and detecting wanted cars. This paper introduced a system that utilizes adaptable components that includes Radio Frequency Identification (RFID) Transponders which could be placed wherever throughout the road then the gathered real-time data are transmitted to the server that stays on static stationary. Moreover, it supports sending Short Message Service (SMS) to closest or nearest police unit or police station via GSM in order to cease the vehicles with violations, and has the capability to inform the driver with the violation, also instant salary payment for the fine thru E-Gov systems (in Jordan). In another study [2], the authors have proposed a car alerting speed limit and crash detection system. The paper introduces a system that uses Micro-ElectroMechanical Systems (MEMS), Radio Frequency (RF), Global Positioning System (GPS) and Global System for Mobile Communications (GSM) technology. The objective of the paper is to design a Smart Display Controller that is designed in a way to suite into the car's dashboard and presents the alerts or warnings of crash and speed limit for the driver. In yet another paper [3], the authors have presented an automatic brake control system of automobile via RFID active tags on the speed limit boards and traffic signals. Their paper proposed a system that is fundamentally based on three stages: identification of RFID transponders (tags), input from the automobile speed sensor into Electronic Control Unit 
(ECU) and eventually, brake control of the automobile. This system utilizes RFID reader (30 m reading range) which sends the reference speed obtained from RFID tag that is linked to either traffic signal or speed limit signs on the road to the ECU unit that will produce an output to control the speed of the car. In alternative study [4], the authors have developed a system that can monitor the traffic jam in real time and the driver is updated through the Short Message Service (SMS) or a web page via Radio Frequency Identification (RFID) and General Packet Radio Service (GPRS) technologies along with Google Maps API. The design of the proposed system was divided into two categories: 1 . the server that generally consists of GSM Modem and web page or portal and 2. The client typically comprises of GPRS Modem and two RFID readers (Texas Instruments). In This system, two Radio Frequency Identification (RFID) readers are located on the road with distance between them and the vehicles are attached with passive RFID tags. So basically the RFID readers will read the passive tags as they pass by them then compute the speed of the vehicle via a running algorithm on the web server which is obtained from the two passing periods of the vehicle based on the tag number that are send by GPRS Modem and the calculated speed is stored inside the data base. Eventually, the driver can determine the traffic jam on the monitored roads using either website or Short Message Service (SMS). The consumer can create a profile in the web page to be automatically informed by traffic situations on certain chosen roads through SMS or E-mail. In another study [5], the authors have introduced a low cost system that manages over speed violations through Zigbee. The system in this paper consists of two sections transmitter section or unit and receiver unit. The transmitter module is located at specific zone, the speed limit is programmed in the PIC 16F877A microcontroller and information is sent to the receiver module which is located inside the car through wireless protocol Zigbee (CC2530), whereas the receiver module is categorized into two units one is placed close to rear view mirror that consists of Zigbee receiver that receives the wireless signal, PIC 16F877A microcontroller that receives the signal from Zigbee receiver then CAN controller that receives the signal sent by microcontroller with data rate of $125 \mathrm{~Kb} / \mathrm{s}$. And the other unit is placed close to the dash board of the vehicle and the communication between two CAN controllers is executed of the two sub-units, in the second sub-unit the CAN controller gets the speed of the car from the speed meter and then transmits it to the microcontroller which in turn compares the speed the car with the speed limit and based on that a conclusion is made. The difference between speeds will be checked continuously and an alert is displayed on Liquid Crystal Display (LCD), moreover a vocal alert is provided to decrease the speed when it's going to exceed the speed limit. In case the driver neglected the warnings, violation is registered and the microcontroller stores all violations experienced by the vehicle and a message containing the speed difference along with information of the car number is sent to traffic police through SMS via GSM modem.

From the previous researches it was observed that systems have addressed certain weaknesses[1], and their paper has presented a reliable, effective and low cost system utilizing both RFID and GSM technology. However, there are still scope for further refinements in the system. The (Phidget USB) RFID readers utilized in the system has a maximum of $10.16 \mathrm{~cm}$ reading range and can't read multiple tags at the same time. Moreover, camera could have been used in the system for evidencing purpose. Hence, the system could use better RFID readers with longer reading ranges along with capability of reading multiple tags and camera to ensure accurate readings and better performance.

Paper [2], has presented a low cost and precise system that uses RFID and GSM technologies to alert the driver about the speed limit on the speed limit zones and to send SMS to traffic police if speed violation occurs or a crash is sensed. On the other hand, a possible limitation pointed out in this system is that in case if the vehicle gets an accident, the MEMS sensor is shook and immediately the longitude and latitude is specified via GPS and is sent to police through SMS. Even though, if the accident occurs inside the house for example the car hits the wall or gate, still the MEMS sensor is shaken and starts the process. However, in case, a camera is placed in the dashboard of the vehicle, a picture could have taken and sent to police along with prior mentioned information (longitude and latitude) to make a decision and see whether this accident has occurred is normal (E.g:, at home) or on the roads.

Study [3], developed a system for traffic management via automatic brake control using RFID technology that is cost effective, flexible and reliable. Electronic Control Unit (ECU) receives the speed of the vehicle from the speed sensor placed in the car and it directly gives a signal to the braking system to control the speed with no prior warning. Hence, transmitting a signal from ECU to a vocal warning device implemented in the vehicle could be further added to the system in case if the driver hasn't noticed the speed limit board or red traffic signal and also scope exists to add camera. The other study [4], introduced a system that is simple, cost effective and efficient to check traffic jam. Similar to previous observations, Global Positioning System (GPS) systems could have added more value as the user is driving will add benefits to the device as well to the users.

From the review, it's clear that the studies are quite similar to each other and on a broader comparison, the study [3], presented a system that controls the vehicle's speed automatically which makes it unique and complex among the rest papers. Further [5] the other study used a different technology for communication between units that is Zigbee wireless protocol which makes it unlike the other introduced systems that are all based on RFID technology. Moreover, [4], developed a system with different concept to manage the traffic by monitoring the traffic jam in real time and update users through SMS or web page via RFID and GPRS technologies. Study [2], designed a system that not only manages speed violation, it also detect crashes which hasn't been discussed by any other referred papers.

Eventually, among all researches and different system, system [1], proposed a system that is excessively similar to the presented project in some aspects, since it utilizes Global System for Mobile Communications (GSM) and Radio Frequency Identification (RFID) technologies. However, their system has implemented short range RFID readers and cannot read various RFID transponders simultaneously which is not a preferable choice for such a system. The presented system will utilize longer range RFID readers with extra characteristic that is a Camera which hasn't been used by any of the previous papers and Short Message Service (SMS). 


\section{SYSTEM FRAMEWORK}
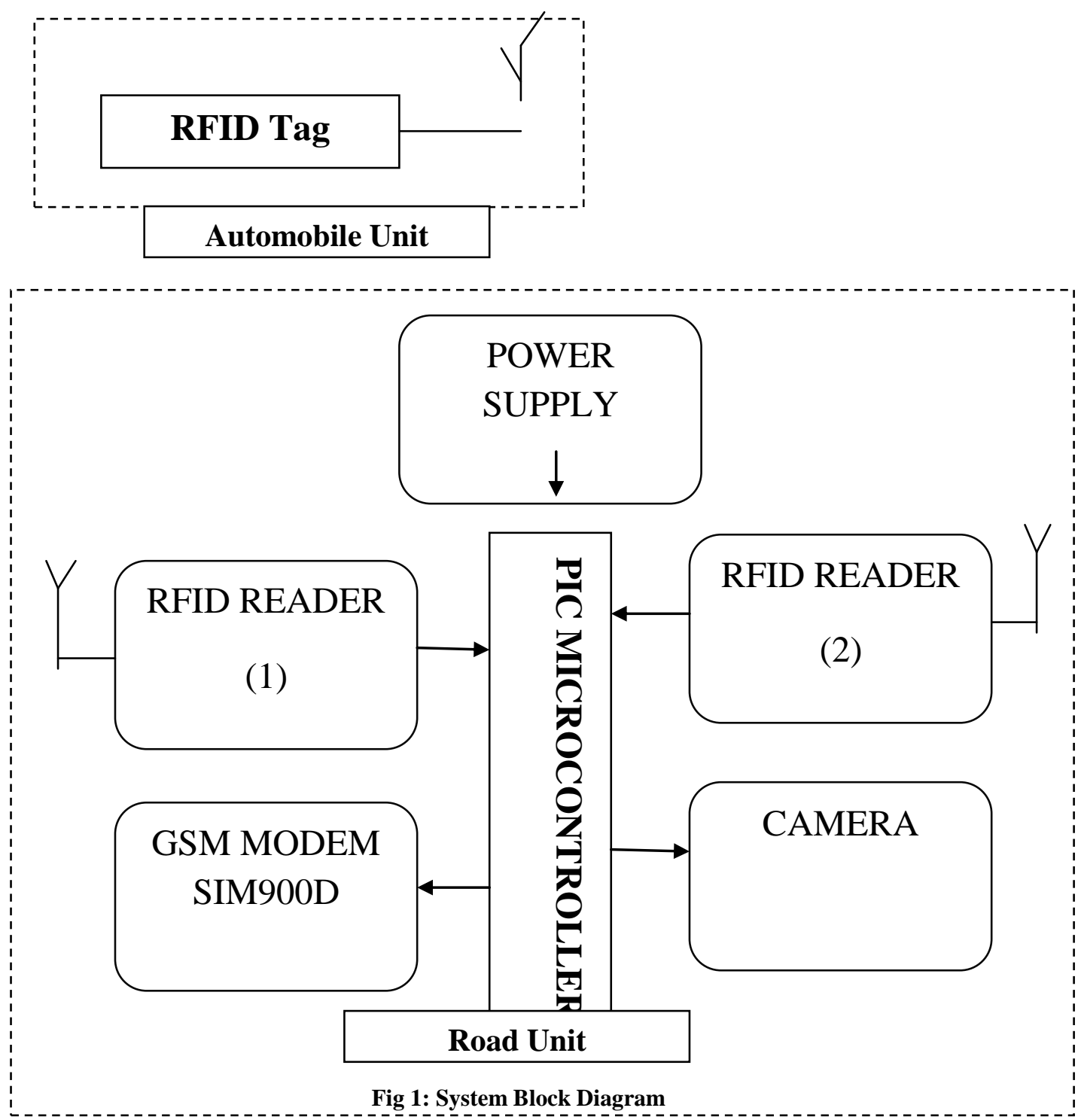

\subsection{Power Supply}

The system is powered by a battery source of $9 \mathrm{~V}$ that is connected to the input pin of voltage regulator (L7805) to get a proper output voltage at the output pin of voltage regulator equal to $5 \mathrm{~V}$ or to step down the voltage from $9 \mathrm{~V}$ to $5 \mathrm{~V}$, which is required for PIC (18F45K22) microcontroller, two RFID readers (ID-20 Innovations) and camera.

\subsection{PIC18F45K22 Microcontroller}

This high performance and low power consumption with XLP technology microcontroller, has 40 pins, five ports (A, B, C, $\mathrm{D}, \mathrm{E})$ and needs power supply $(2.3 \mathrm{~V}$ to $5.5 \mathrm{~V})$. The main characteristics of this microcontroller is that it has two Enhanced Universal Synchronous Asynchronous Receiver Transmitter (EUSART) ports that are essentially required for connecting two ID-20 Innovations (RFID) and GSM/GPRS SIM 900D Module to the microcontroller, large program memory of $32 \mathrm{~KB}$, EEPROM data memory of 256 bytes, SRAM data memory of 1536 bytes and an improved core for high speed operation.

\subsection{SIM900 GSM/GPRS Module}

This cost effective Quad-band GSM/GPRS Module (wireless modem) is specifically manufactured in a way that it can both appear and perform with a small dimensions of $33 \times 33 \times 3$ mm like any cell phone with its own number by accepting the SIM card of any GSM network operator. It delivers communication services such as voice calls, sending Short Message Service (SMS), fax and Multimedia Messaging Service (MMS) through mobile network via connecting the SIM900D Modem to a microcontroller or computer, the offered services by SIM900D module are charged by the GSM network operator and requires instructions that are known as AT commands to control the modem and execute functions. With its low power consumption GSM SIM900D Module is employed for sending SMS that contains about the automobile's owner along with location where speed violation has taken place to both Royal Oman Police (ROP) and vehicle's owner to inform about the speed violation once it's committed. 


\subsection{Radio Frequency Identification Module}

Radio Frequency Identification (RFID) system consists of Reader with antenna and transponders (tags) that consists of microchip where data is stored and antenna, it uses Radio Frequency (RF) waves for communication and transferring data between RFID reader and tag. In this project the two readers used to emit radio waves are active readers in order to activate the passive tag (no power supply needed and cheaper) that is embedded into the vehicle's plate number that will return its code. In this project, two simple RFID reader modules are employed from ID innovations (ID-20 Innovations) that produce a radio frequency electro-magnetic field with a frequency of $125 \mathrm{KHz}$ (Low Frequency Band) for reading and also writing data with a maximum distance of 16 $\mathrm{cm}$. It requires $5 \mathrm{~V}$ power supply and has dimensions of $38 \mathrm{x}$ $40 \times 7 \mathrm{~mm}$.

\subsection{Camera}

Camera is a sophisticated further feature to the system that will provide a clear evidence of committing speed violations on the roads by the automobiles once it receives a signal to take a snap of the vehicle from the microcontroller using a 5
$\mathrm{V}$ relay which is basically a switch the functions electrically and then the pictured will be stored in SD card.

\subsection{System Working Principle}

The proposed system utilizes RFID readers (ID-20 Innovations), GSM/GPRS SIM900D module where a SIM card is inserted and camera which are interfaced to the microcontroller (PIC19F45K22). Based on the RFID passive tag placed into the vehicle as it passes by the first RFID reader where the information of the vehicle stored in the tag is extracted, the count-down timer within microcontroller is fixed to specific time according to the distance between two RFIDs and the speed limit will be activated and in case the vehicle's tag is read by the second RFID reader before timer reaches zero, then the microcontroller recognizes that a violation has occurred by this certain tag and then a signal via microcontroller is sent to both camera to take a snap and GSM/GPRS SIM900D modem to send SMS to the number of the owner of the vehicle acquired from the tag and Police to inform about the committed speed violation.

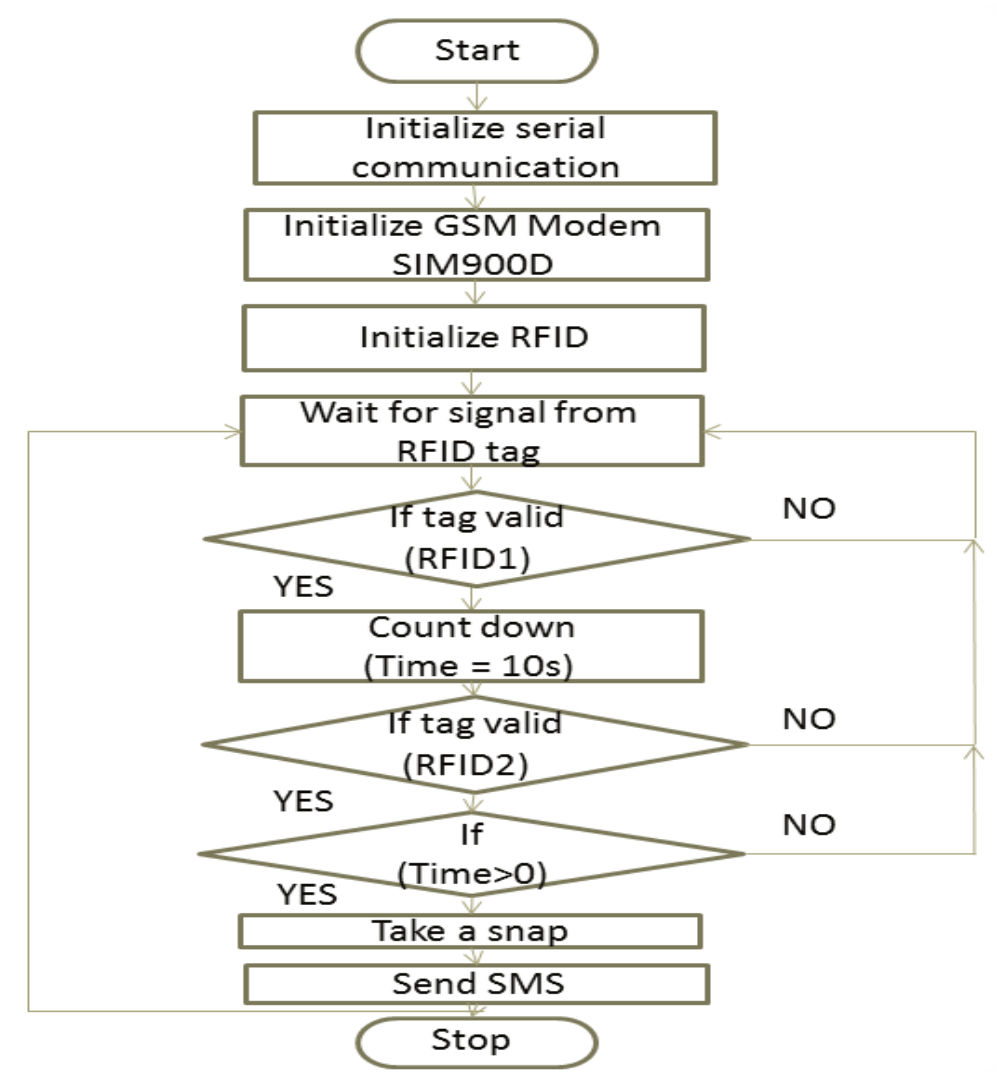

Fig 2: System Flow Chart

\section{DESIGN SIMULATION AND RESULTS}

The circuit was successfully designed as per the block diagram via Proteus v7.10 with all the components that are required for the proposed system. The two virtual terminals are used for displaying the output and input responses between the microcontroller and external devices (RFID reader and GSM modem) since the GSM/GPRS SIM900D module and RFID reader (ID-20 Innovations) components are not available in Proteus. Also, a camera is not available in Proteus so it was replaced by LED (Light Emitting Diode), so LED will glow to indicate the flash of the camera. All the required tasks were executed correctly. 


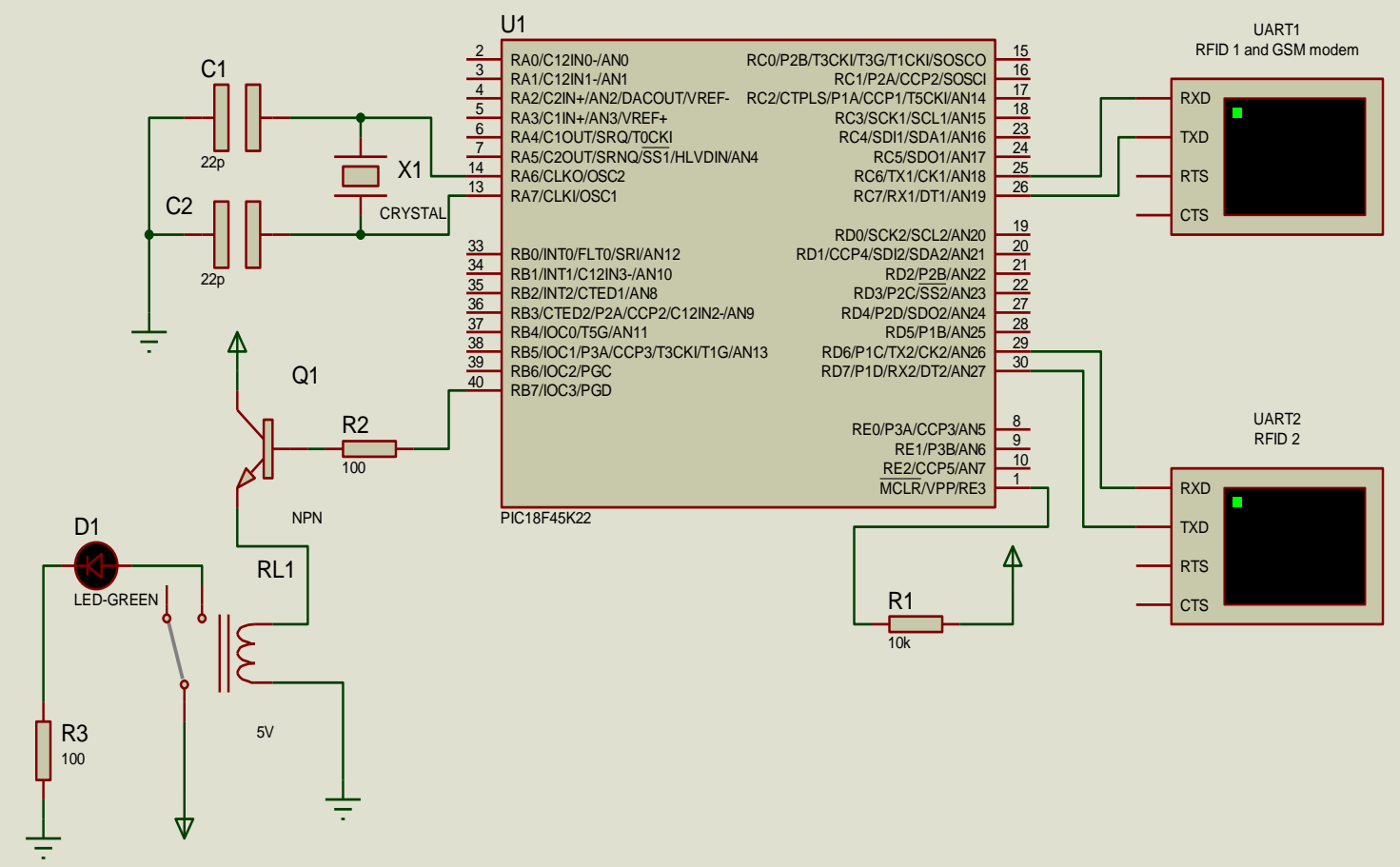

Fig 3: System Model

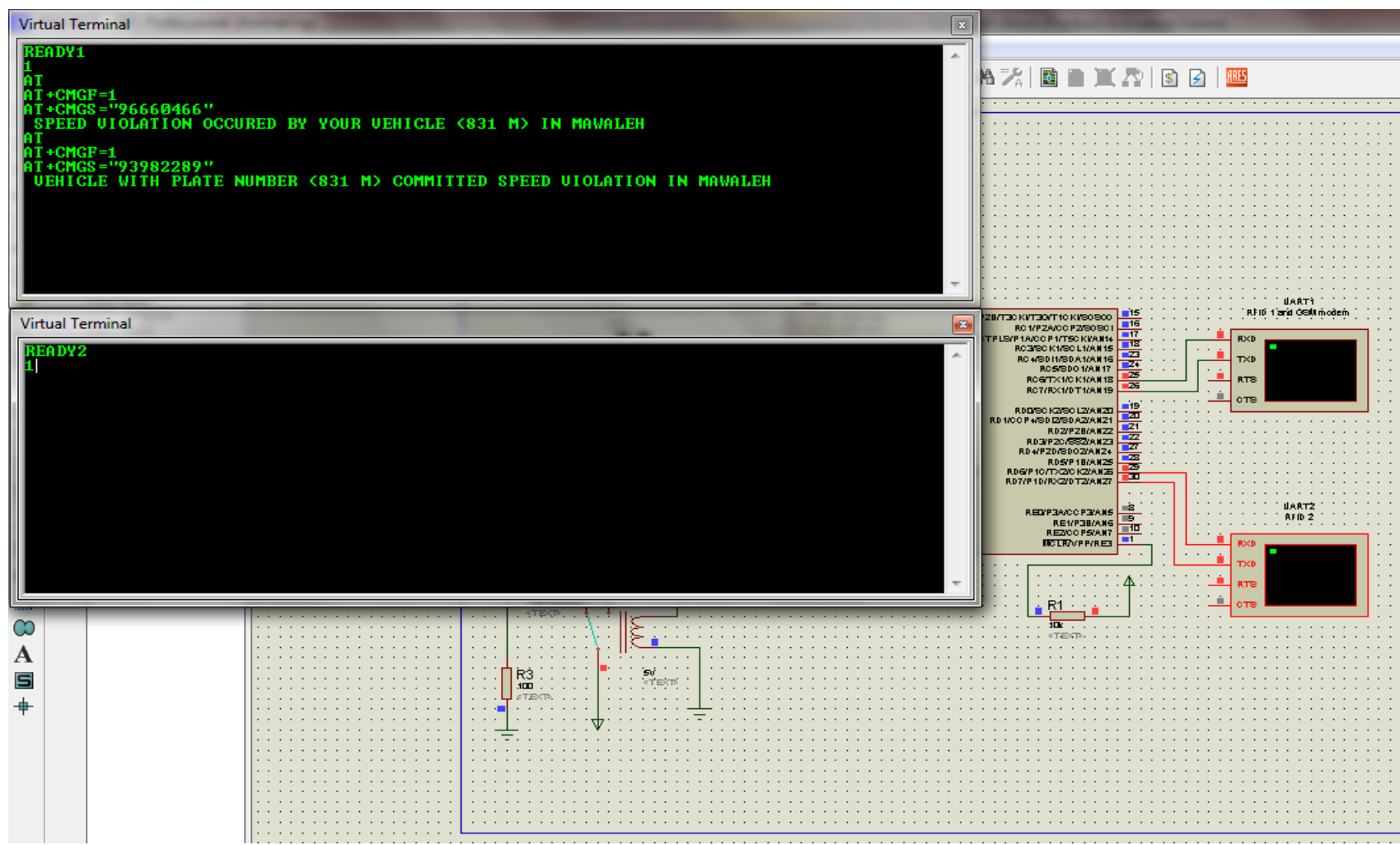

Fig 4: Simulation Results 


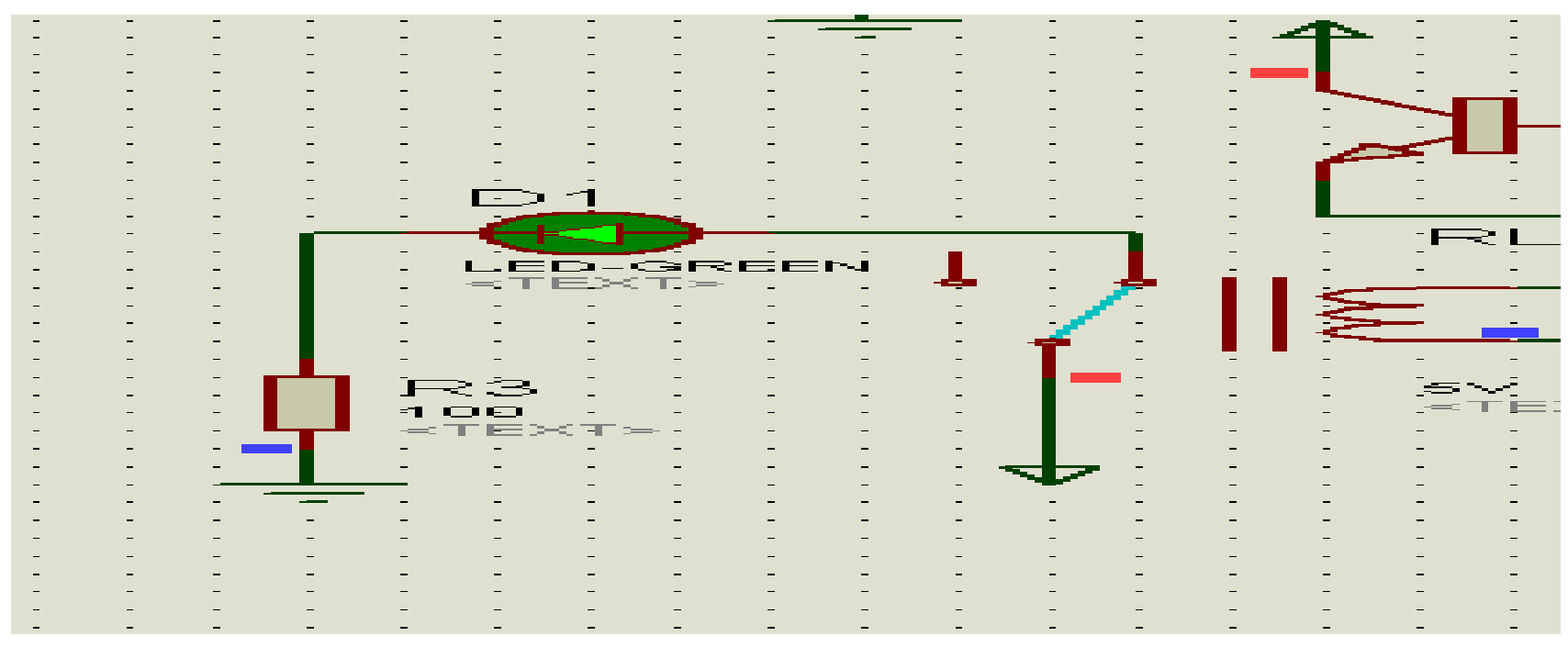

Fig 5: LED Glowing Indicating the Flash of Camera

The executed system was found to be performing or functioning as per expectations. If the automobile crosses the distance between two RFIDs before time reaches zero then the SMS was successfully sent to both numbers (ROP and the owner), also the LED glowed indicating as if a snap of the vehicle has been taken.

\section{CONCLUSION}

This paper presents a cost effective, accurate, flexible system to help in preventing over speeding violations, based on the literature survey and observing different traffic management systems with using diverse technologies and components. Generally, each system was divided into two units a transmitter unit and a receiver unit where one is place on the monitored road and the other attached to the automobile. Various technologies and protocols were utilized for several systems such as; Radio Frequency Identification (RFID), Zigbee, Global System for Mobile Communications (GSM), Global Positioning System (GPS), General Packet Radio Service (GPRS), etc. Based on the introduced system the transmitter unit is fixed on the road using RFID technology and the Receiver unit is attached to the automobile's plate number and communication between them occurs through radio waves, and GSM technology is used for notifying purpose. The paper delivered explanation of the circuit diagram of the introduced system. The project circuit diagram was designed via Proteus v7.1 for software simulation. Moreover, a prototype of the proposed system was assembled with the required components on bread board initially for testing then on Printed Circuit Board (PCB). The system provided efficient and accurate results. As a future work, the actual system would be implemented shortly.

\section{ACKNOWLEDGMENTS}

The author would sincerely love to dedicate her special appreciations and thankfulness exclusively to whoever supported directly and indirectly for their continued encouragement, assistance and proper guidance with patience throughout the project.

\section{REFERENCES}

[1] Hiasat, R. H. and Almomani, A. A. 2013. Real Time Radio Frequency Identification Vehicles Data Logger Traffic Management System. Journal of Emerging Trends in Computing and Information Sciences, (Feb. 2013), Vol. 4, No. 2.

[2] Singh, N. and Teja, R. 2013. Vehicle Speed Limit Alerting and Crash Detection System at Various Zones. International Journal of Latest Trends in Engineering and Technology, (Jan. 2013), Vol. 2, No. 1.

[3] Thirukkovulur, A. K., Nandagopal, H. and Parivallal, V. 2012. Intelligent Vehicle Control Based on Identification of Road and Traffic Signal Operated RFID transponders: International Conference on Advances in Electrical and Electronics Engineering (ICAEE). (2012), 339-343, Penang, Malaysia.

[4] Aloul, F. et al. GuideME: An Effective RFID-Based Traffic Monitoring System. Sharjah: American University of Sharjah.

[5] Rubini, R. \& Makeswari, A. U. 2013. Over Speed Violation Management of A Vehicle Through Zigbee. International Journal of Engineering and Technology, (Mar. 2013), Vol. 5, No. 1.

[6] Singh, K., Chawla, R. and Singh, H. 2014. Intelligent Speed Violation Detection System. International Journal of Engineering and Technical Research, (Jan. 2014), Vol. 2, No. 1.

[7] Hajeb, S. et al. Traffic Violation Detection System Based on RFID. World Academy of Science, Engineering and Technology, (Feb. 2013), Vol. 7.

[8] Rappaport, T. 2003. Wireless Communications: Principles and Practice, New York: Prentice Hall, Second Edition. 